\title{
Heart Rate Detection from the Supratrochlear Vessels using a Virtual Reality Headset integrated PPG Sensor
}

\author{
Michal Gnacek \\ Bournemouth University \\ United Kingdom \\ mgnacek@bournemouth.ac.uk \\ Ellen Seiss \\ Bournemouth University \\ United Kingdom \\ eseiss@bournemouth.ac.ukk
}

\author{
David Garrido-Leal \\ Universitat de Barcelona \\ Spain \\ dagale.95@gmail.com
}

Theodoros Kostoulas
Bournemouth University
United Kingdom
ostoulas@bournemouth.ac.uk

\author{
Ruben Nieto Lopez \\ Universitat de Barcelona \\ Spain \\ rub1993rose10@gmail.com
}

Emili Balaguer-Ballester
Bournemouth University
United Kingdom
eb-ballester@bournemouth.ac.uk

\author{
Ifigeneia Mavridou \\ Emteq Ltd. \\ United Kingdom \\ ifi@emteq.net
}

\author{
Charles Nduka \\ Emteq Ltd. \\ United Kingdom \\ charles.nduka@emteq.net
}

\begin{abstract}
An increasing amount of virtual reality (VR) research is carried out to support the vast number of applications across mental health, exercise and entertainment fields. Often, this research involves the recording of physiological measures such as heart rate recordings with an electrocardiogram (ECG). One challenge is to enable remote, reliable and unobtrusive VR and heart rate data collection which would allow a wider application of VR research and practice in the field in future. To address the challenge, this work assessed the viability of replacing standard ECG devices with a photoplethysmography (PPG) sensor that is directly integrated into a VR headset over the branches of the supratrochlear vessels. The objective of this study was to investigate the reliability of the PPG sensor for heart-rate detection. A total of 21 participants were recruited. They were asked to wear an ECG belt as ground truth and a VR headset with the embedded PPG sensor. Signals from both sensors were captured in free standing and sitting positions. Results showed that VR headset with an integrated PPG sensor is a viable alternative to an ECG for heart rate measurements in optimal conditions with limited movement. Future research will extend on this finding by testing it in more interactive VR settings
\end{abstract}

\section{CCS CONCEPTS}

- Human-centered computing $\rightarrow$ Virtual reality; • Hardware $\rightarrow$ Digital signal processing.

Permission to make digital or hard copies of all or part of this work for personal or classroom use is granted without fee provided that copies are not made or distributed for profit or commercial advantage and that copies bear this notice and the full citation on the first page. Copyrights for components of this work owned by others than the author(s) must be honored. Abstracting with credit is permitted. To copy otherwise, or republish, to post on servers or to redistribute to lists, requires prior specific permission and/or a fee. Request permissions from permissions@acm.org.

ICMI '20 Companion, October 25-29, 2020, Virtual event, Netherlands

(c) 2020 Copyright held by the owner/author(s). Publication rights licensed to ACM.

ACM ISBN 978-1-4503-8002-7/20/10 . \$ \$15.00

https://doi.org/10.1145/3395035.3425323

\section{KEYWORDS}

Virtual reality, PPG, ECG, heart rate, affective neuroscience

\section{ACM Reference Format:}

Michal Gnacek, David Garrido-Leal, Ruben Nieto Lopez, Ellen Seiss, Theodoros Kostoulas, Emili Balaguer-Ballester, Ifigeneia Mavridou, and Charles Nduka. 2020. Heart Rate Detection from the Supratrochlear Vessels using a Virtual Reality Headset integrated PPG Sensor. In Companion Publication of the 2020 International Conference on Multimodal Interaction (ICMI '20 Companion), October 25-29, 2020, Virtual event, Netherlands. ACM, New York, NY, USA, 5 pages. https://doi.org/10.1145/3395035.3425323

\section{INTRODUCTION}

Virtual reality (VR) has found its way into mainstream research and its use is steadily increasing due to numerous benefits, such as precise control over stimuli, ability to display objects in 3 dimensions [17] and increased ecological validity [12] to name a few. Many studies have quantified the affective responses to VR environments using biosensors $[1,9,15,18]$. ECG measurements, via individual sensors on the chest or integrated in a belt set-up, have been a standard practice for heart rate measurements [2]. However, an ECG device in addition to the VR setup, can be cumbersome in a typical VR research lab setting because the ECG device must be put on separately, and a researcher needs to ensure correct and consistent positioning of the sensors. Some studies have explored the idea of using and visualising emotions for enhanced user behavior analysis [6] which integrated sensors could help facilitate. ECG measures electrical signals produced by the heart with each contraction through electrodes placed on the skin. PPG sensors detect blood volume changes by measuring the amount of light absorbed or reflected by the skin. This changes throughout the cardiac cycle due to the pressure pulse of blood being pumped by the heart. Our team in collaboration with Emteq [10] explored the integration of PPG sensors into a soft mask that can be used as an insert for a commercial VR headset. The careful integration of the PPG sensor into the VR headset, reduces the strenuous positioning of additional devices for heart rate data collection, which is 
prone to human error, making the setup process significantly less complicated for the experimenter. This process could reduce the sensor set-up time required per participant and enhance the overall user-experience, by limiting obtrusiveness of additional sensors on the skin of the wearer.

The Covid-19 pandemic of 2020 caused major disruptions to research-intensive institutions and forced a transition to remote working model [13]. Remote data collection might be the only feasible option for many researchers in near future. With standard ECG devices, equipment setup would have to be done by the participant with advised virtual oversight from the experimenter [7] but this can lead to misplacement of electrodes which can cause reduced signal-to-noise ratio as well as data misinterpretation due to signal distortions [5]. This ease of use of a VR headset with integrated PPG sensor could be of a great benefit to researchers working with any VR application where heart data needs to be collected. It would also enable remote heart rate data collection where previously, obtrusive devices requiring technical understanding, not be practical in a remote setting had to be used.

\section{RELATED WORK}

Although not often used in affective neuroscience and VR research, PPG is a well-established, low cost and non-invasive method of collecting heart rate measures (e.g. beats per minute (bpm)). PPG sensors are already in common use today in medicine, research, athletic monitoring and everyday personal devices [4]. New applications for this technology such as early diagnosis of cardiovascular disease are continuously being identified [3]. PPG sensors are most effective when placed on specific parts of a human body (finger, earlobe and forehead). This is due to the different characteristics and distribution of blood vessels that are used by light emitting sensors which measure changes in light absorption. The biggest challenge for the reliable recording of heart rate is the reduction of noise caused by motion artefacts. The forehead has a rich blood supply from supratrochlear and supra-orbital arteries and veins. This, plus the relatively stable platform afforded by the underlying boney structure and the thinness of the skin, could help reduce these artefacts [16]. Researchers have identified methods for detecting segments in the signals where motion artefacts are present by using data obtained from a motion sensor and by taking advantage of slow changes in statistical values of heart rate features used to exclude outliers [14]. PPG signal processing and feature extraction have been continuously optimised with the aim of identifying most optimal filters and pre-processing steps under different conditions [8]. An earlier version of the mask used in this study was previously reported in a study which investigated the ability to detect arousal levels with ECG and PPG when participants watched affective and neutral videos [11]. Findings from that study indicated that arousal discrimination was slightly worse with the PPG sensor compared to the ECG device. That study, however, did not analyse heart rate (bpm) for each signal. Instead, it focused on arousal classification from derived metrics. The aim of the current study was to directly compare the accuracy of heart rate measurement (in bpm) collected by the ECG belt and a PPG sensor located at a new improved position (supratrochlear vessels), which was integrated into the EmteqPro VR headset.

\section{EXPERIMENTAL SETUP}

This experiment aimed to establish the feasibility of replacing commonly used ECG devices like a chest belt for heart rate monitoring with a VR headset integrated PPG sensor. This study has built on previous work by using a new, higher resolution PPG sensor, an improved fit of the mask and a better PPG sensor location.

\subsection{Participants}

For this study, 21 participants were recruited (14 males, 6 females). Of those, four participants were excluded due to noisy data and one because the recording duration was too short. The remaining 16 participants had a mean age of 25.81 years (SD: 6.4, range: $19-$ 43 years). Participants filled in a short demographics survey and a survey to screen for mental or physical conditions, as well as any medications that could affect heart rate levels. The study was conducted in accordance to the Declaration of Helsinki and all participants signed a consent form having read an information sheet.

\subsection{Equipment}

3.2.1 Headset with mask. Figure 1 shows the latest iteration of Emteq's EmteqPro v1.0 system attached to HTC VIVE VR headset. It featured EMG, PPG and IMU sensors. This system comes with proprietary software which allows synchronous streaming and data recording onto a PC via Bluetooth.

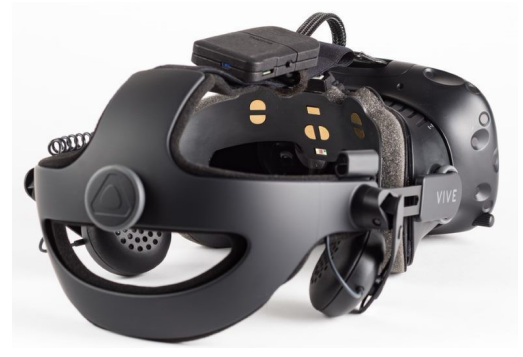

Figure 1: EmteqPro v1.0 attached to HTC VIVE headset.

3.2.2 ECG Belt. Custom-built ECG belt worn by participants around their chest (upper abdomen area) for ECG data collection at a sampling rate of $1000 \mathrm{~Hz}$ (same refresh rate as PPG made direct comparison easier) to a PC via Bluetooth.

3.2.3 PPG sensor. Commercially available $1000 \mathrm{~Hz}$ pulse oximeter and heart rate sensor from OSRAM Opto Semiconductors $\mathrm{GmbH}$. It is designed and optimised for wearable and mobile device.

\subsection{VR environment}

Figure 2 shows a screenshot of the virtual space used for data collection. It consists of a dark environment with a sun rising on the horizon, with grid lines around the floor and no sound. It is part of the SteamVR ecosystem and is used as a default menu and loading screen. No controllers were used by participants. This environment did not include any animations or interactable objects to minimise participants response and allow for baseline data to be captured. 


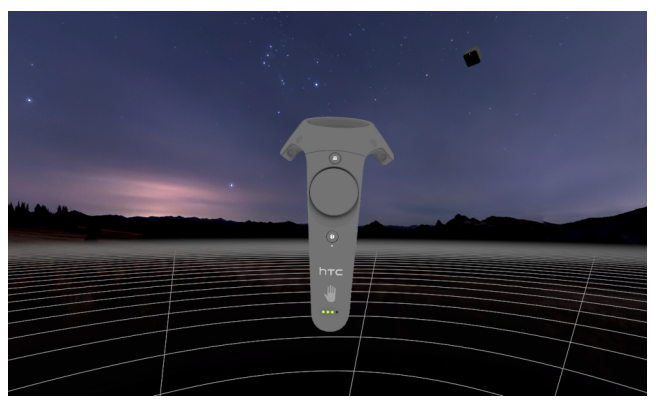

Figure 2: SteamVR default lobby (image lightened).

\subsection{Procedure}

Once the equipment had been securely placed on each participant, the signal quality of both sensors was assessed by looking at the real time signal and their fit adjusted until quality was satisfactory. No controllers were used for this data collection and participants could place their hands wherever felt most comfortable. Participants were instructed to try and relax, act naturally but try to move as little as possible and look directly ahead. Participants were then asked to sit comfortably in an office chair at which point, data started being recorded. After just over one minute, participants were asked to stand up while still trying to keep a neutral facial expression and look straight ahead. Data while standing was also recorded for just over one minute. Once data collection has finished, participants were asked to rate their arousal, valence, and stress levels using a 9-point rating scales after the experience.

\section{ANALYSIS}

\subsection{Signal processing}

4.1.1 ECG. Butterworth highpass filter (4th order, cutoff: $0.7 \mathrm{~Hz}$ ) was used to filter out any noise below the minimum heart rate. Butterworth lowpass filter (3rd order, cutoff: $10 \mathrm{~Hz}$ ) was used to filter out frequencies where electrical and muscle artefacts and noise are likely. The first 3 seconds and the last 1 second were removed to avoid filtering artefacts. Then, heart rate peaks were detected using a filtered signal 1st order differential.

4.1.2 PPG. A more restrictive Butterworth lowpass filter (6th order, cutoff: $5 \mathrm{~Hz}$ ) was used to try and filter electrical and movement artefacts. The first 3 seconds and last 1 second were removed to avoid filtering artefacts. Signal was detrended by subtracting the best straight-fit line to emphasise features, and a moving average with time window of $10 \mathrm{~ms}$ was applied to the smooth out the signal.

\section{RESULTS}

As expected, subjective ratings (1-9) collected for 13 out of 16 participants indicated low arousal $(\mathrm{M}=2.38, \mathrm{SE}=0.33)$, neutral valence $(\mathrm{M}=4.38, \mathrm{SE}=0.27)$, and low stress levels $(\mathrm{M}=1.84, \mathrm{SE}=0.19)$ during the recordings. There was no correlation between subjective ratings and any of the heart rate measures. Moderate correlation between arousal and stress was found, $\mathrm{p}=.585$. The signal-to-noise ratio was visually inspected for the PPG and ECG data. Peaks were easily identifiable in 16 selected participants, out of 21 participants. Baseline experiment was divided into sitting and standing. The findings in Figure 3 show means \pm SE for these two positions, and the combined total. Results from Shapiro-Wilk normality tests of ECG and PPG beats per minute showed that all measures were normally distributed ( $p>0.05$ for all conditions). Kendall's tau-b correlation coefficients showed that ECG and PPG signals are highly correlated for the combined, sitting and standing conditions, with $\mathrm{p}<.000$ for each condition. When comparing ECG heart rates for the sitting $(\mathrm{M}=72.8 \mathrm{bpm}, \mathrm{SE}=2.86)$ and standing $(\mathrm{M}=85.6 \mathrm{bpm}, \mathrm{SE}=3.07)$ conditions, participants' heart rate was significantly increased by $12.8 \mathrm{bpm}$ in the standing condition, $\mathrm{t}(15)=4.94, \mathrm{p}=<.000$. Heart rate comparisons for the PPG measurements were similar. Again, when comparing the sitting $(M=73.29, S E=2.88)$ and standing $(M=85.32$, $\mathrm{SE}=3.12$ ) conditions, the heart rate in the standing condition was significantly increased by $12.02 \mathrm{bpm}$ compared to the sitting condition, $\mathrm{t}(15)=4.36, \mathrm{p}=.001$.

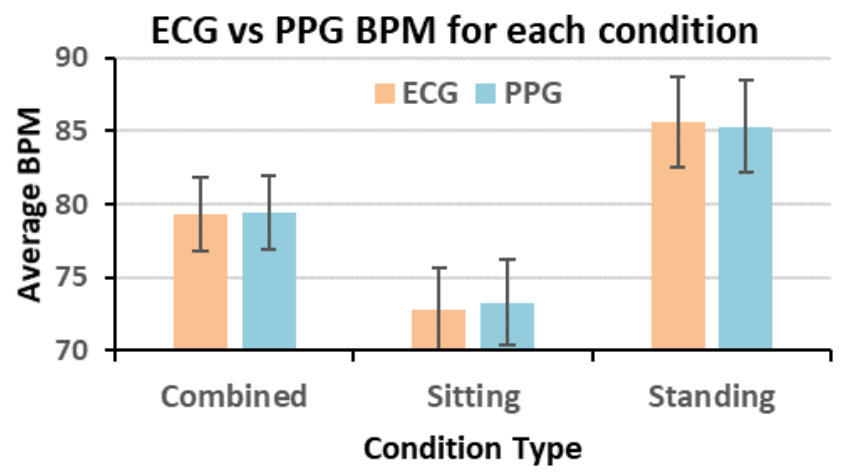

Figure 3: Heart Rate (bpm) measurements with ECG and PPG sensors.

Additionally, ECG and PPG signals for both conditions were divided into time segments of 10 seconds with a 5 second sliding window. Data was divided with this method into 8 separate sections. The same filtering, peak detection and BPM algorithms were used as described above to calculate heart rates (bpm) for each window separately. Figures 4 and 5 show mean heart rates for each time window. A repeated measures ANOVA with the factors recording methods (ECG vs PPG), condition (sitting vs. standing) and time window (1-8) revealed no significant difference between the ECG and PPG methods in heart rate measures, $\mathrm{F}(1,15)=.010, \mathrm{p}=.923$. Sitting vs standing condition were significantly different $F(1,15)$ $=19.79, \mathrm{p}<.001$. Finally, the ability to detect a difference between sitting and standing did not significantly differ between recording methods, $\mathrm{F}(1,15)=4.45, \mathrm{p}=.052$.

\section{DISCUSSION AND CONCLUSIONS}

The aim of the current study was to verify the ability to reliably detect heart rate changes with a PPG sensor embedded in a VR headset. Results indicate that, in optimal conditions, a PPG sensor can effectively replace a standard ECG belt, as heart rate measures were almost identical for both methods. Strong correlation between PPG and ECG signals was found, confirming the viability of the 


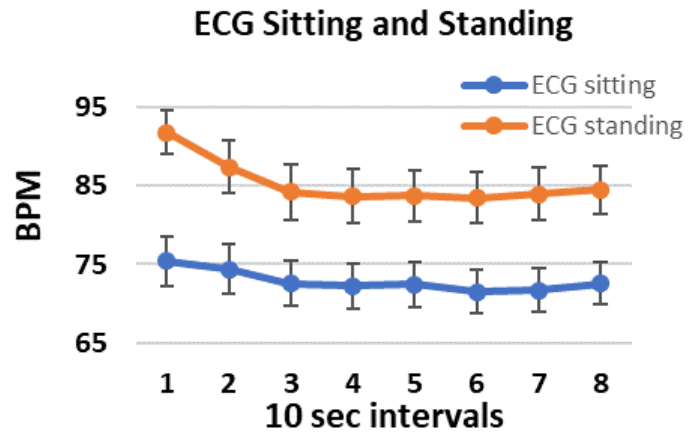

Figure 4: Mean ECG BPM values for sitting and standing.

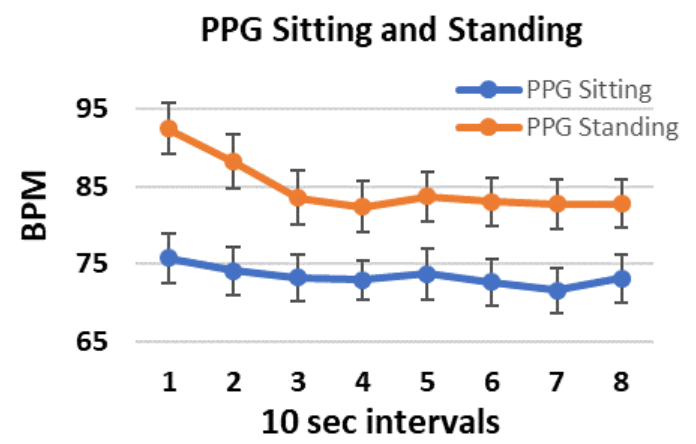

Figure 5: Mean PPG BPM values for sitting and standing.

used hardware and software. Both signals showed the expected significant increase in heart rate (bpm) going from a sitting to a standing position, confirming the viability of both methods to detect heart rate variations with changing positions. Both recording methods were equally sensitive to this change (Figure 3 ). The same was true when sliding time windows of 10 seconds duration were used. Again, heart rates did not significantly differ between the ECG and PPG recording methods and between the sitting and standing conditions (Figures 4 and 5). In the standing condition, heart rates visibly increased initially to then slowly reduce over time. This can be explained by the initial increase in a metabolic demand caused by the action of standing up and thus increasing the heart rate momentarily. Once the metabolic demand has been satisfied and the body has acclimatised to the new standing position, heart rate settled down to the new baseline. However, this approach has some limitations. Firstly, PPG sensors do pick up more noise when the device does not fit snugly on the face, as evidenced by the number of participants that had to be excluded. Secondly, when looking at individual time windows, some participants were found to have elevated heart rates in the PPG compared to ECG signal, usually explained by movement artefacts that were not present in the ECG. PPG heart rate values quickly returned to the ECG equivalent once the noise has subsided, i.e., usually within the next 10 second time window. Non randomised order of the two conditions (sitting then standing) could have potentially affected standing results due to habituation. Studies with a balanced gender participant pool as opposed to our male favouring gender distribution bias, could potentially report different BPM values due to the differences in the cardiac functions between the genders. Lastly, the environment used in this study had neutral valence, low arousal and low stress levels as intended for the purpose of baseline data collection, which leads us to the future work section.

\section{FUTURE WORK}

The aim of the study was to investigate whether it is possible to replace obtrusive ECG devices with a PPG sensor built into a VR headset. Results have shown that when good quality PPG signal is captured, it is a very reliable method of obtaining heart rate measures. However, the quality of the PPG signal degrades under conditions when the device does not fit snugly on the face or when the user moves excessively. The susceptibility of the PPG sensors to motion artefact is well recognised. Indeed, even devices such as the Apple Watch do not measure heart rate during vigorous motion, with measurement only taken when the inertial sensor detects low amplitude movements. It was important to establish optimal operational conditions for measuring high quality facial PPG signals before moving on to more interactive VR experiences, involving walking. Movement artefacts occurring in these interactive experiences require additional processing such as filtering and outlier removal in order to achieve reliable heart rate measurements. Since this study was performed in 2019, the facial interface has been already redesigned to optimse the sensor position and allow for different face sizes to reduce motion artefact. In addition, accelerometer data collected by the IMU sensor integrated into the mask can be used to detect periods of increased movement to help identify motion artefacts. Data captured by the included PPG proximity sensor (how close is the sensor to the face of the user) can also provide insights into which parts of the data are likely to be unreliable. Poor quality signal could be avoided by monitoring real time data during the recording. In addition, an automated signal monitoring process could let users know about the problem to enable them to adjust the fit. This continuous monitoring could greatly increase the reliability of the PPG signal and solve the problem of having to exclude a proportion of participants. Any studies requiring the collection of heart rate in VR could benefit from this approach as it can be easily integrated into VR headsets without having to add obtrusive ECG devices. Health monitoring is another potential application as the amount of time we spend in VR continues to increase. Consistent and reapeated use over a long period of time could provide an insight into long term health changes. Adaptive VR experiences where environmental parameters are changed in response to user's heart rate would benefit greatly by being able to accurately detect this data directly from the headset. Easy access to heart rate features aids the development of multimodal affect recognition models for VR, where different physiological signals are used in combination together to determine user's affective state.

\section{ACKNOWLEDGMENTS}

This work is supported by Bournemouth University and Emteq Ltd. via the Centre for Digital Entertainment (EPSRC Grant No. $\mathrm{EP} / \mathrm{L} 016540 / 1)$ 


\section{REFERENCES}

[1] Imtiaj Ahmed, Ville Harjunen, Giulio Jacucci, Niklas Ravaja, and Michiel M. Spapé 2017. Total immersion: Designing for affective symbiosis in a virtual reality game with haptics, biosensors, and emotive agents. Lecture Notes in Computer Science (including subseries Lecture Notes in Artificial Intelligence and Lecture Notes in Bioinformatics) 9961 LNCS, 23-37. https://doi.org/10.1007/978-3-319-57753-1 3

[2] Piero Fontana, Neusa R.Adão Martins, Martin Camenzind, René M. Rossi, Florent Baty, Maximilian Boesch, Otto D. Schoch, Martin H. Brutsche, and Simon Annaheim. 2019. Clinical applicability of a textile 1-lead ECG device for overnight monitoring. Sensors (Switzerland) 19 (6 2019). Issue 11. https: //doi.org/10.3390/s19112436

[3] Mohammad Ghamari. 2018. A review on wearable photoplethysmography sensors and their potential future applications in health care. International fournal of Biosensors \& Bioelectronics 4 (2018), 195. Issue 4. https://doi.org/10.15406/ijbsbe. 2018.04.00125

[4] S. M.Riazul Islam, Daehan Kwak, Md Humaun Kabir, Mahmud Hossain, and Kyung Sup Kwak. 2015. The internet of things for health care: A comprehensive survey. IEEE Access 3 (6 2015), 678-708. https://doi.org/10.1109/ACCESS.2015. 2437951

[5] Michał Kania, Hervé Rix, Małgorzata Fereniec, Heriberto Zavala-Fernandez, Dariusz Janusek, Tomasz Mroczka, Günter Stix, and Roman Maniewski. 2014. The effect of precordial lead displacement on ECG morphology. Medical and Biological Engineering and Computing 52 (2 2014), 109-119. Issue 2. https //doi.org/10.1007/s11517-013-1115-9

[6] Daniel Kepplinger, Günter Wallner, Simone Kriglstein, and Michael Lankes. 2020 See, Feel, Move. Proceedings of the 2020 CHI Conference on Human Factors in Computing Systems, 1-14. https://doi.org/10.1145/3313831.3376401

[7] Yue Li, Lu Zheng, and Xuewen Wang. 2019. Flexible and wearable healthcare sensors for visual reality health-monitoring. Virtual Reality \& Intelligent Hardware 1 (8 2019), 411-427. Issue 4. https://doi.org/10.1016/j.vrih.2019.08.001

[8] Yongbo Liang, Mohamed Elgendi, Zhencheng Chen, and Rabab Ward. 2018 Analysis: An optimal filter for short photoplethysmogram signals. Scientific Data 5 (5 2018). https://doi.org/10.1038/sdata.2018.76

[9] Javier Marín-Morales, Juan Luis Higuera-Trujillo, Alberto Greco, Jaime Guixeres, Carmen Llinares, Enzo Pasquale Scilingo, Mariano Alcañiz, and Gaetano Valenza. [n.d.]. Affective computing in virtual reality: emotion recognition from brain and heartbeat dynamics using wearable sensors OPEN. ([n. d.]). https://doi.org/ 10.1038/s41598-018-32063-4

[10] Ifigeneia Mavridou, James T. McGhee, Mahyar Hamedi, Mohsen Fatoorechi, Andrew Cleal, Emili Ballaguer-Balester, Ellen Seiss, Graeme Cox, and Charles Nduka. 2017. FACETEQ interface demo for emotion expression in VR. Proceedings - IEEE Virtual Reality, 441-442. https://doi.org/10.1109/VR.2017.7892369

[11] Iigeneia Mavridou, Ellen Seiss, Theodoros Kostoulas, Charles Nduka, and Emili Balaguer-Ballester. 2018. Towards an effecve arousal detecon system for virtual reality. Proceedings of the Human-Habitat for Health (H3): Human-Habitat Multimodal Interaction for Promoting Health and Well-Being in the Internet of Things era - 20th ACM International Conference on Multimodal Interaction, ICMI 2018, 1-6. https://doi.org/10.1145/3279963.3279969

[12] G Menshikova, Yu Bayakovski, E Luniakova, M Pestun, and D Zakharkin. [n.d.]. Virtual Reality Technology for the Visual Perception Study.

[13] M. Bishr Omary, Jeetendra Eswaraka, S. David Kimball, Prabhas V. Moghe, Reynold A. Panettieri, and Kathleen W. Scotto. 2020. The COVID-19 pandemic and research shutdown: Staying safe and productive. , 2745-2748 pages. Issue 6. https://doi.org/10.1172/JCI138646

[14] David Pollreisz and Nima TaheriNejad. 2019. Detection and Removal of Motion Artifacts in PPG Signals. Mobile Networks and Applications (8 2019), 1-11. https: //doi.org/10.1007/s11036-019-01323-6

[15] Felix Schoeller, Philippe Bertrand, Lynda Joy Gerry, Abhinandan Jain, Adam Haar Horowitz, and Franck Zenasni. 2019. Combining Virtual Reality and Biofeedback to Foster Empathic Abilities in Humans. Frontiers in Psychology 9 (2 2019), 2741. Issue FEB. https://doi.org/10.3389/fpsyg.2018.02741

[16] Toshiyo Tamura, Yuka Maeda, Masaki Sekine, and Masaki Yoshida. 2014. Wearable Photoplethysmographic Sensors-Past and Present. Electronics 3 (4 2014), 282-302. Issue 2. https://doi.org/10.3390/electronics3020282

[17] Christopher J Wilson and Alessandro Soranzo. 2015. The Use of Virtual Reality in Psychology: A Case Study in Visual Perception. (2015). https://doi.org/10. 1155/2015/151702

[18] Peter Zeile and Bernd Resch. 2018. Combining Biosensing Technology and Virtual Environments for Improved Urban Planning. GI Forum 1 (2018), 344-357. https://doi.org/10.1553/giscience2018_01_s344 